\title{
ETIMOLOGIE ROVIGNESI
}

L'Autrice passa in rassegna 11 parole rovignesi : bàcolo "trabaccolo", bascaràn "pesce al di sotto dei due etti", bruièlo "brolo, frutteto", bugiadàga "pacchia", buriòn "tuono", paladiàna " pietra o legno in ornato posta ai lati del portale di un palazzo", panceîro "popone vernino", parlanceîn "parolaio", pastanàcia "pastinaca", priviàl "piviale", pulpulòn "polpaccio", fissandone l'etimo e integrando, in tal modo, le ricerche etimologiche di studiosi precedenti quali M. Doria e F. Crevatin.

Questo articolo, costituisce una sorta di seguito a quanto già da me pubblicato, in AMSIA 1996', dove, appunto, trattavo dei seguenti termini rovignesi (desunti dal dizionario del dialetto rovignese di Antonio e Giovanni Pellizzer): fulpià v. tr. "pestare" (da considerarsi pressocché identico al friulano folpeâ "calcare, sgualcire calcando" (così NP 330, Faggin I 459) e al triestino polipiar "tastare (figurativo), saggiare il terreno (di un avversario), nella terminologia dei giocatori di tressette" (GDDT 431), tutti dal latino *PALPIDIĀRE "palpeggiare", con dissimilazione a distanza, per il rovignese e il friulano, di $p-p>f-p$, come per il triestino e il veneto giuliano folpo (ittion.) "polipo" (Rosamani 389), dal latino POLYPUS); incinàse v. rifl. "inchinarsi, piegarsi" (da latino INCLINĀRE, con paralleli qua e là nei vari dialetti italiani, ad esclusione del veneto); maseîdio s. m. "eccidio, sterminio" (deformazione di italiano omicidio, cfr. per l'aferesi l'italiano micidiale); patruòma s.f. "pesantezza di stomaco, groppo, blocco" (parallelo all'italiano antico e al triestino patùrnia "accesso di malinconia (o di nausea)" (GDDT 979): aggiungiamo, a completamento di quanto qui esposto, la variante, sempre rovignese patoûgna, ib. Pellizzer 671); pudarà v.tr. "appoderare" e quindi "possedere, entrare in possesso" (cfr. il derivato pudaramènto s.m. "capacità, facoltà, possibilità", Pellizzer 720); pulseîn s.m. "pulcino" (anche del dignanese, più vicino, lessicalmente al tipo toscano - e francese (poussin) - che non al veneto pulișin); sanbutà v.i. "cicalare, chiacchierare a lungo, cianciare" (con riscontri col gradese zambotà, col bisiacco sanbotar, col veneto periferico zambotar, (cfr. Pellizzer 809, Prati EV 151, Domini 395, Rosamani 922, 1242), cfr. però anche l'italiano ciambolare, ant. ciambottare); saniciàre s.f.pl. "passeri", costituisce l'anello di congiunzione, che finora mancava, tra il derivato saniciareîn, anche sarniciareîn, "passero" e il francese chante-claire, da cui trae origine tutta una piccola famiglia lessicale per la designazione di quest'uccello); sanpièrla s.f. "mano o piede spropositati", anche "ciabatta", connettibile col tipo triestino zanfarda (impiegata con gli stessi due significati della

1 M. R. Cerasuolo Pertusi, Storie di parole rovignesi, AMSIA vol. XCVI della Raccolta (XLIV della Nuova Serie), Trieste 1996, pp. 617-625. 
parola rovignese), ital. ant. cianfarda "sorta di veste antica" (GDDT 796), piuttosto che con zampa); servato s.m. "quercia" (da un originario *CERRUUS, ampliamento di CERRUS "cerro" (una sorta di quercia, ben presente dalle nostre parti), da cui dendronimi vari e toponimi in Friuli ed Istria, cfr. Rosamani 935, 1008, 1253 e G. Frau in "Misc. G. Mastrelli Anzilotti", Firenze 1992, pp. 184-185); șinșièga s.f. "baldoria, molta allegria, mangiare e bere ", collegabile col tipo triestino ganzega ("scampagnata con bisboccia", pressoché identico, anche per significato, GDDT 260), con l'isolano ganșèga ("gruppo di gente allegra, festa vivace e alla buona", Vascotto 122), dal tardo latino *GAUDIĀTICA (< GAUDEO "godo", cfr. Bondardo 80), attraverso una dissimila-zione-assimilazione a distanza $g-s-g>s-s-g$ ); squatrinà v.t. "spiare, tener d'occhio" (ampliamento del tipo italiano squadrare nel significato di "osservare attentamente, scrutare da capo a piedi", cfr. DELI V 3607 s.v. squatra); ulivài s.m.pl. "mutuo, prestito" (da un livel "canone, interesse", lat. *LIBELLUS o meglio AD LIBELLUM, con $-a(d)$ o $a(b)>\mathrm{u}$ come in rovignese umòndo "molto", Pellizzer 1082), (dal lat. ABUNDE, cfr. M. Doria, AMSIA 1994, p. 370). Per la riprova di tutto ciò rimando a F. Semi, Glossario, p. 224, il quale cita testualmente per Pirano (a. 1305), la locuzione "ad fictum sive libellum".

Benché non intitolati come monografie etimologiche specifiche, segnalerò, ancora, i contributi di due illustri studiosi di linguistica istriana: le pagine della prefazione al Pellizzer-Pellizzer di Franco Crevatin e una recensione di M. Doria (AMSIA 1994), il quale coglie l'occasione di tratteggiare la storia della stratificazione lessicale del rovignese, distinguendo fra prestiti recentissimi (dal triestino, dal croato, dall'inglese, dal tedesco, dallo spagnolo) e prestiti - talvolta molto antichi, es. bacarà "gozzovigliare" dal veneziano, e parole che possono definirsi del vecchio fondo dialettale preveneto, tali surùra "sorella", linbùro "sporgenza del tetto" (da gr. EMPÓRION "piazza, spiazzo"); antichi certo anche cașàl nel significato di "casa cadente, diroccata" e il già citato umòndo "molto" (da ABUNDE, con concordanze col gradese e, soprattutto, col friulano). Ma il Doria si era occupato, anche in altre occasioni di etimi rovignesi (e istrioti in generale, v. note bibliografiche avanti).

Rimarchevoli, come si è detto, anche le note etimologiche abbozzate dal Crevatin nella prefazione citata; come ad es. per balantròn "groviglio, viluppo, intrigo" (da gr. LABYRINTHOS), balbigà o balbagà "vacillare, camminare oscillando" (da BALBICĀRE id.), mașido "ammansito, sottomesso, umile" (dal lat. MANSUĒTUS), paladù$r a$ anche paladoûra "roncola" (dal lat. PUTATŌRIA).

Questo mio contributo è certo più modesto di quelli sopra elencati, non pertanto spero riesca a gettare un po' di luce sulla storia di questo importante, caratteristico dialetto.

Avverto, preliminarmente, che anche in questa seconda serie di voci (tratte dalle lettere B e P del succitato dizionario) si ricava l'impressione di un'estrema eterogeneità, soprattutto se riferita alla fase più recente, direi contemporanea, del dialetto. Infatti, solo due delle voci da me indagate rivelano un certo carattere di arcaicità e sono quindi assegnabili al vecchio fondo dialettale preveneto (esse sono, nella fattispecie, pri- 
viàl "piviale" e pulpulòn "polpaccio"). Qualche interesse per la storia lessicale rovignese (ed istriana in generale) paiono offrire bruòlo "piccolo orto, frutteto" e forse anche buriòn "tuono" (se questo è raccostabile a 'bora'). Altre parole sono adattamenti più o meno vistosi di termini della lingua letteraria (tali paladiàna "pietra o legno in ornato posta ai lati del portale di un palazzo" e parlanceîn "parolaio, detto di persona verbo-sa") o sono, come capita spesso, venetismi più o meno camuffati (es. bàcolo "trabaccolo"). Di minor conto le conclusioni che si possono trarre da panceiro "popone vernino", mentre pastanàcia "pastinaca", che sia pure debolmente, pare legato al Friuli (ma non per il - ča!).

1. bàcolo s.m. - trabaccolo. Gli AA. non tentano alcun etimo. Certo è, comunque, che la parola non ha nulla a che fare con l'omofono triestino bàcolo "blatta, scarafaggio". Questo ricorre, veramente, come prestito anche nel rovignese, ma sotto la forma, lievemente adattata, bàculo (p. 81). Data una certa propensione del dialetto rovignese all'aferesi (qualche esempio significativo di tale procedimento lo trovia-mo in Doria, AMSIA 1994, p. 372, es. caco "macaco", ossia "sciocco", e anche smònica "fisarmonica"), non esito a vedere in bàcolo un abbreviamento del veneziano (del triestino ecc.) trabàcolo "trabaccolo, trabiccolo" (per il suo etimo e diffusione v. GDDT 749 s.v.). L'etimo è accettabile anche se il rovignese, in aggiunta a bàcolo, conosce anche le forme complete trabàcolo e trabàculo (Pellizzer vol. II, 1054 s.v.).

2. bascaràn s.m. "Nel linguaggio dei pescatori il pesce al di sotto dei due etti". Gli AA. poi precisano che l'Ive menziona questa parola anche per il vecchio dialetto (che si suppone fosse anch'esso di tipo istrioto come i vicini gallesanese e sissanese) di Pola: "dinotare quella quantità di pesce che si muove al di sopra". Collegato ad esso risulta il plurale bascaràmi "i pesci che non fanno parte della massa, del banco" ("pesce misto" v. un po' avanti). Evidentemente si tratta di una voce uscente al singolare con un $-m$ - passata a $-n$. La considererei volentieri un derivato da PǏSCULUS "piccolo pesce", da cui proviene l'antico pisano pèscolo "briciolo", il lucchese piscolo "minuzzolo, bruscolo" (sull'acqua, nel vino) e simm. (così DEI IV 2875) e, soprattutto, il bellunese pessùcola (da *PISCULU, però rifatto su PISCIS > 'pesse', su cui REW(S) 6532). Ad esso si aggiunse il suffisso di collettivo italia-no -ame (cfr. rottame, sartiame, o anche gradese novelame "pesce novello", Cor-batto 186) ed -l- come più volte in rovignese, si sarà poi rotacizzato. Possibile, però, pensare anche a un PISCĀRE o 'pesca' più il suffisso composto-olame (cfr. in triestino e gradese barcolame "insieme di piccole barche"), sempre con rotacismo. Per lo scambio $p / b$ cfr. baciro/pancéiro (su cui v. avanti).

3. bruièlo s.m. - brolo, frutteto. Attestato anche (v. avanti) bruòlo, il quale concorda con la forma, più frequentemente attestata in Istria, brolo, che ritroviamo ad es. 
ad Albona, a Parenzo, a Buie, a Cittanova e a Salvore, soprattutto come toponimo. Ma a Parenzo è attestato, come toponimo (a. 1325) Broilo e l'appellativo bròilo è anche del pisinese (Rosamani 119 s.v., cfr. la scrivente CES, AMSIA 1990, p. 190), quest'ultimo più vicino al tipo friulano bròili. Tornando al nostro bruièlo, le voci ad esso più vicine sono, nell'antico veneziano Brogio (su cui v. Prati EV p. 25) e il toponimo triestino antico Brojet (V. Scussa p. 23 Cam.) o Broglietto (Ireneo della Croce p. 199) o Bruiet (P. Cancellieri "il Giovane", s. XIV ex., ed. M. Szombathely POr. N. S. 2,1966 , p. 261), oggi Broletto, ma, fin dal ' 400 anche, metatizzato, Burieto (a. 1401, Cavalli TS ‘400, p. 345). Broilo, brolo e broglio sono tre evoluzioni distinte a partire da una forma originaria (di origine celtica) BROGILO, o direttamente da questa (e questo vale, oltre che per broilo anche per il fiulano bròili) o attraverso un francese breuil (v. REW 1324, ma sono possibili anche altre spiegazioni, cfr. REW(S)), da cui, appunto, broio, brogio, broglio e infine, con depalatalizzazione, brolo. Come si constata, sul suolo istriano hanno fatto presa tutte e tre le varianti ed è questo uno dei sintomi della frammentazione dialettale e della complessità delle stratificazioni lessicali della penisola (per un altro caso, riguardante il nome dell"'edera" v. Doria AMSIA 88, 1988, p. 289 nonché in "Festschr. Muljačič", Hamburg 1987, p. 262). Tant'è vero che anche nell'ambito di una stessa area, magari molto ristretta, si sovrappongono più forme, come succede, appunto, per Trieste, per Parenzo; per Capodistria (dove si alternano Brol(l)o a. 1348 , Broylio a. 1423, Broglio a. 1556, Brolio a. 1538. Ancora oggi, Piazza del Brolo e, su scala minore, come ora ci accorgiamo, a Rovigno.

4. bugiàdaga s.f. - pacchia. E' un derivato di bui "bollire", con acquisizione di un tratto fonetico squisitamente venezianeggiante (tipo Buge "Buie", Vertenegio "Verteneglio"), piuttosto raro nel lessico del rovignese, comunque attestato (cfr. giarsìra "ieri sera", giàgia < cr. jaje "uova"). In altre parole, partiamo da un BULLIĀTICA "brodo" (cfr. con suffissazione un po' diversa il francese bouillon "brodo" o bouille "pappa, poltiglia"). Per il suffisso -ĀTICUM cfr. ad es. l' italiano companàtico (dial. companàdego), per il semantismo il ben noto modo di dire (andare in) brodo di giùggiole.

5. buriòn s.m. - tuono. La voce non può certo definirsi isolata. Essa ricorre, infatti, anche nell'orserese (Rosamani 132): inoltre potrebbe identificarsi con l'omofono bisiacco buriòn "bora impetuosa", cui si accompagna la variante buriùn "bora a folate riccorrenti". E' facile ammettere che dal significato originario di "vento impetuoso" si sia passati a quello di "tempesta accompagnata da vento", a "tempesta", "temporale" in genere e, infine, a "tuono". A detta del Rosamani, infatti, l'istriano burion significa anche "burrasca improvvisa", come a dire che il legame con le voci più settentrionali è assicurato. Non solo, l'isolamento della voce del rovignese è infranto, anche, dalla comparsa nel medesimo del verbo burià "tuonare" 
(che Pellizzer-Pellizzer 155, registrano due volte!). Sono tutte voci che, nel loro insieme, si dovrebbero far risalire al latino BOREA (o meglio *BORIA). Dato però che *BORIA potrebbe conservare il suo - $i$ - 'etimologico' unicamente in dominio linguistico friulano, ci si domanda se la voce istriana - anziché essere un lontano prestito dal friulano - non sia, piuttosto, una derivazione locale, autonoma, da bora con suffisso -ILLIŌNEM, tipico dell'istrioto: (cfr. dign. siòn "uccello", Rosamani 1033, o la forma con l'affricata zeiòn, Supplemento al Dalla Zonca di M. Debeljuh 338, fradion "fratello", su cui v. M. Doria "IL" 5, 1979, p. $112 \mathrm{e}$ chi scrive, CES, p. 198; cfr. però, nello stesso campo semantico, anche il francese tourbillon), e che si abbia a che fare con un'omofonia casuale. In tal caso burià sarà un retrogrado da questa forma nominale (e andrebbe tenuto d'occhio, quale ne sia l'etimo, anche il lessotipo buriana).

6. paladiàna s.f. - pietra o legno in ornato posta ai lati del portale di un palazzo. Nessuna indicazione etimologica da parte degli AA. del Dizionario, ma è oltremodo evidente trattarsi del femminile sostantivato dell'aggettivo italiano palladiano "relativo all'architetto Palladio". E basterà confrontare, anche se non perfettamente coincidenti col nostro, le seguenti voci registrate nel GrDLI : palladiana "pavimento palladiano" (costituito da pietre e lastre di marmo di forma irregolare, unite da materiale cementante rosso) e finestra palladiana "finestra a forma di lunetta divisa da elementi portanti ecc.", anche capriata di tipo palladiano.

7. panceîro s.m. - popone vernino (cucumis melo hibernus). Neppur questa è voce isolata. Essa si identifica facilmente col ben noto triestino (e anche capod., parent., zar., nonché friul., muglis. e bis.) baciro, (su cui v. GDDT s.v.) e, soprattutto col dign. bacèiri (Dalla Zonca 10). Quanto alla consonante iniziale, il nostro panceîro si ricopre con le forme attestate a Muggia e a Chioggia (v. GDDT Suppl. 842) nonché a Venezia stessa (qui accanto a bachiri [Boerio 54]).

La voce è di ultima origine orientale, probabilmente persiana (bakūra "frutto primaticcio"), giuntoci attraverso Venezia (o la Dalmazia); per baćîr o bacîr nel cr. di Arbe, di Cherso e di Ragusa (quivi anche bačir) v. P. Skok ERHiS s.v.e, in precedenza, M. Deanović in "Omagiu Rosetti", Bucarest 1965, p. 158). E in vista di quest'ultimo canale di penetrazione colgo l'occasione di ricordare la macchietta spalatina "Toma Bacir", con "il suo testone di ebete galleggiante, come i palloncini colorati nelle fiere, sopra un corpicciattolo da fiaba", così F. Bettizza "Il Meridiano" 7-111991 p. 32. Per baciro, fig. "testa" cfr. anche M. Doria in "Studi Deanović" (=BALM 22-28, 1980-86, p. 71), per Baciro soprannome a Pola, Rosamani 53, s.v.

8. parlanceîn s.m. - parolaio. L'etimo dall' italiano parlantino “id." (XVII sec.) è fuori discussione e non meriterebbe in questa sede approfondirlo se non per alcune considerazioni di cronologia relativa ai mutamenti fonetici tipici dell'istrioto. Det- 
ta parola costituisce, infatti, un prezioso indizio riguardo l'epoca della dittongazione di $-i$ - in -éi- (sia a Rovigno che a Dignano). Essa non solo è posteriore al recepimento di questo italianismo, ma posteriore anche alla palatalizzazione "istriana" (cfr. cos'cera "costiera" passim) davanti ad $i$ o $i$. Se la dittongazione di cui sopra fosse un fatto antico, essa avrebbe bloccato la palatalizzazione, palatalizzazione, dunque, che si pone, cronologicamente, tra l'assunzione del prestito e la dittongazione (buona ultima, ripetiamo, in questa serie di fenomeni linguistici).

9. pastanàcia s.f. - pastinaca (Pastinaca sativa, L.). Non occorre, anzi non è nemmeno necessario, scomodare, per spiegare questa forma, il friulano. Il caso parrebbe, di primo acchito, analogo a quello di maruòcia "fondo, deposito che lasciano l'olio e il vino", evidentemente identico al tipo veneto maroca (per questo termine e i suoi vari significati v. GDDT s. v.), dove il - ča finale (da etimologico $-k k a$ ) è spiegabile unicamente per influsso friulano. Pastinàcia, invece, presuppone o un latino volgare *PASTINĀCIUM (cfr. le forme tipo ital. pasticciano, elencate in DEI IV 2788) o, forse, meglio *PASTINĀCULA, ricostruibile, possibilmente, anche con l'aiuto di forme parallele friulane (su cui v. da ultimo Pellegrini-Zamboni FPF II N. 159). Nel caso si volesse optare per la prima alternativa, il rovign. pastinàcia sarebbe, quindi, un italianismo (toscanismo), se collegabile con le voci friulane, d'ambito italo-settentrionale.

10. priviàl s. m. - piviale. Priviàl non è "corruzione" di (ital.) piviàle(e), bensì una continuazione locale diretta di lat. mediev. (a. 1125, Sella 448) di pluviale s.n. "mantello per ripararsi dalla pioggia durante le processioni" (cfr. a. franc. plovial, pluiel "Regenmantel" REW 6621, lucch. pievale e irp. kjovę(j)ale id. REW(S), ital. ant. pieviale, Boccaccio, pioviale a. 1365 e ploviale s. XV, DEI IV 2961. Per pluviale in testi medievali della nostra regione cfr. a Trieste a. 1358 (D. Bloise ATr. 40, 1980, p. 56) "quattuor pluvialia (em. Doria: plumalia Cod.) ad usum sacerdotalem, quae pluvialia (cod. plumalia) debeant servire divino culto et perpetuo remanere cathedrale ecclesie Tergesti"). La dissimilazione $l-l$ in $r-l($ o $l-r)$ non va certo considerata fenomeno inaudito in ambito rovignese: basterebbe rimandare alla voce pruòlaga "proroga", elencata nel nostro Dizionario a pagina 716. Quindi priviàl, ripeto, potrebbe considerarsi forma locale, non necessariamente legata a quella di altri dialetti (veneto compreso) che avrebbero potuto influire ma non l'hanno fatto sul rovignese.

11. pulpulòn s.m. - polpaccio. Già registrato nel Rosamani 837 e in GDDT (s.v. pùpola), non è stato, forse, adeguatamente sfruttato ai fini lessicali e nei suoi rapporti col dignan. pulpulòn "id.". Ambedue rappresentano un notevole arcaismo rispetto alle forme del veneto pùpola (Boerio 540) o pùpula, dove la prima $l$ è caduta per aplologia dissimilativa e che si può definire forma standard, diffusasi, oltre che 
nel triestino, anche in friulano (pùpule), nel polese pùpole (B. Manzini, AMSIA 90,1990, p. 283) e anche nel vallese pùpola (Cernecca 87), pur essendo questo un dialetto "istrioto" (però molto venezianizzato!). Si rammenti comunque che a Grado, Marano e nel capodistriano pùpola, -ula significano "polpastrello" e non "polpaccio" e che non è possibile, allo stato attuale delle ricerche, precisare quale dei due significati sia il più antico. L'etimo è, ad ogni modo, evidente: lat. PULPA + suffisso di diminutivo (aggiunto posteriormente) -OLUS. Da questa breve disanima possiamo concludere che *pùlpula, -òn rappresentino, possibilmente, un arcaismo del solo istrioto, anche se, doverosamente, occorre avvertire che i tratti conservativi non sono di per sé indizi sufficienti di caratterizzazione dialettale (nessuno ci garantisce, poi, del fatto che in un non lontano domani, capiti di trovare attestato in dominio veneto una forma del tipo pulpula, e che quindi, da questo punto di vista, preveneto d'Istria e veneto si debbano collocare sullo stesso piano).

\section{Abbreviazioni bibliografiche}

AMSIA

Atti e Memorie della Società Istriana di Archeologia e Storia Patria, Parenzo-Venezia-Trieste.

$\mathrm{ATr}$

Archeografo Triestino, Trieste.

BALM

Bollettino dell'Atlante Linguistico Mediterraneo, Venezia-Roma.

BOERIO

G. Boerio, Dizionario del dialetto veneziano ${ }^{2}$, Venezia 1856.

BONDARDO

M. Bondardo, Dizionario etimologico del dialetto veronese, Verona 1986.

CAVALli TS ' $400 \mathrm{~J}$

Cavalli, Commercio e vita privata di Trieste nel 1400, Trieste 1910.

Cerasuolo CES

M. R. Cerasuolo Pertusi, Il contributo dell'etimologia alla storia della neolatinità istriana, AMSIA 90, 1990, pp. 187-251.

CERNECCA

D. Cernecca, Dizionario del dialetto di Valle d'Istria, Trieste-Fiume 1986.

CORBaTro

A. Corbatto, Vocabolario della parlata gradese, Grado 1995.

Dalla ZonCA

G. A. Dalla Zonca, Vocabolario dignanese-italiano, a cura di M. Debeljuh, Fiume-Trieste 1978.

DELI

Dizionario etimologico della lingua italiana, di M. Cortelazzo e P. Zolli, Bologna 1979-1988.

DOMINI

S. Domini, A. Fulizio, A. Miniussi, G. Vittori, Vocabolario fraseologico del dialetto "bisiàc", Bologna 1985. ERHiS

P. Skok, Etimološki rječnik hrvatskoga ili srpska jezika (4 voll.), Zagabria 1971-1974.

Faggin

G. Faggin, Vocabolario della lingua friulana, 2 voll., Udine 1985. 
M. Doria - C. Noliani, Grande dizionario del dialetto triestino, Trieste 1987.

GrDLI

G. Battaglia, Grande dizionario della lingua italiana, Torino 1961- .

IL

Incontri Linguistici, Trieste-Udine.

IRENEO

P. Ireneo della Croce, Historia antica e moderna, sacra e profana della città di Trieste, Venezia 1698. NP

Il Nuovo Pirona - Vocabolario friulano, Udine 1935.

PELLEGRINI-ZAMBONI FPF

G. B. Pellegrini-A. Zamboni, Flora Popolare Friulana, Udine 1966.

Pellizzer-Pellizzer

POr

A. e G. Pellizzer, Vocabolario del dialetto di Rovigno d'stria, Trieste-Rovigno 1992.

\section{PRATI EV}

REW

A. Prati, Etimologie venete, Venezia-Roma 1968.

W. Meyer-Lübke, Romanisches Etymologisches Wörterbuch, Heidelberg 1935. REW(S)

P. A. Farè, Postille italiane al Romanisches Etymologisches Wörterbuch di W. Meyer-Lübke,... Milano 1962. ROSAMANI

E. Rosamani, Vocabolario giuliano, Bologna 1958. SCUSSA

V. Scussa, Storia cronografica di Trieste dalla sua origine fino all'anno 1695, a cura di F. Cameroni, Trieste 1863.

SEMI SELLA

F. Semi, Glossario del latino medioevale istriano, Venezia 1990.

P. Sella, Glossario latino italiano, Città del Vaticano 1944.

VASCOTTO

A. Vascotto, Voci della parlata isolana nella prima metà di questo secolo, Imola 1987.

\section{Povzetek}

\section{ROVINJSKE ETIMOLOGIJE}

$\mathrm{V}$ prispevku je obravnavanih 11 rovinjskih besed, in sicer: bàcolo 'trabakula, dalmatinska in istrska ladja $\mathrm{z}$ dvema jamboroma', bascaràn 'majhna riba, z manj kot 20 dag teže', bruièlo 'sadovnjak', bugiàdaga 'obilje, ugodje', paladiàna 'kamnit ali lesen steber z ornamenti ob portalu kake palače', panceîro '(zimska) melona', parlanceîn 'gobezdalo', pastanàcia 'pastinak, rebrinec (Pastinaca sativa)', priviàl 'pluvial, liturgično oblačilo', pulpulòn 'meča'. Avtorica ugotavlja etimologijo teh izrazov in tako dopolnjuje etimološke raziskave, ki sta jih zastavila Mario Doria in Franco Crevatin. 